\title{
A InTervenção Do Enfermeiro De Reabilitação Na Promoção DA ACESSIBILIDAde
} La Intervención De Los Enfermeros De Rehabilitación En La Promoción De La AccesibiLidad Arquitectónica

\section{The Role Of Rehabilitation Nurses To The Architectural Accessibility Promotion}

\author{
Rute Salomé Da Silva Pereira1; Maria Manuela Martins1 ; Bárbara Gomes"; \\ Jose Alberto Laredo Aguilera²; João Santos ${ }^{1}$
}

1 - ESEP; 2 - Universidad de Castilla-La Mancha

\section{RESUMO}

Enquadramento: os enfermeiros de reabilitação são decisivos na promoção de ambientes acessíveis pela eliminação de barreiras arquitetónicas para a reintegração das pessoas com mobilidade condicionada.

Objetivos: Compreender se a eliminação de barreiras arquitetónicas são uma preocupação dos enfermeiros.

Metodologia: estudo quantitativo, descritivo, exploratório. Técnica de amostragem não probabilística acidental em bola de neve, constituída por 56 enfermeiros. A colheita de dados efetuou-se através de um questionário ad hoc de autopreenchimento recorrendo ao formulário GOOGLE, em Novembro de 2016.

Resultados: $60,7 \%$ dos enfermeiros não sabe a quem se dirigir para eliminar barreiras arquitetónicas e 58,9\% não desenvolve nenhum tipo de intervenção nesse sentido. Verificamos diferenças significativas favoráveis aos enfermeiros de reabilitação em relação a deterem mais conhecimento sobre: legislação específica para a acessibilidade $(p=0,000)$ e, a quem se dirigir para solicitar a eliminação de barreiras arquitetónicas na comunidade $(p=0,024)$.

Conclusão: a acessibilidade e a eliminação de barreiras arquitetónicas devem fazer parte da prática diária dos enfermeiros.

Descritores: enfermagem em reabilitação; estruturas de acesso; pessoas com deficiência; limitação da mobilidade

\section{RESUMEN}

Marco contextual: Los enfermeros de rehabilitación son decisivos en la promoción de espacios accesible mediante la eliminación de barreras arquitectónicas para la integración de las personas con movilidad reducida.

Objetivo: Comprender si la eliminación de barreras arquitectónicas son una preocupación de los enfermeros

Metodología: estudio cuantitativo, descriptivo, exploratorio mediante un muestreo no probabilístico accidental en bola de nieve, con una muestra de 56 enfermeros. Se aplicó un cuestionario ad hoc de auto-llenado con recurso al formulario GOOGLE, en noviembre de 2016.

Resultados: $60,7 \%$ de enfermeros no saben a quién se deben dirigir para eliminar barreras arquitectónicas, el $58,9 \%$ no realiza ningún tipo de intervención en este sentido. Se observa diferencias significativas a favor de enfermeros de rehabilitación que tienen mayor conocimiento sobre: legislación específica para la accesibilidad $(p=0,000)$ y a quién deben de dirigir la solicitud de eliminación de barreras arquitectónicas en la comunidad $(p=0,024)$.

Conclusión: La accesibilidad e la eliminación de barreras arquitectónicas deben ser una parte de la práctica diaria de los enfermeros.

Descriptores: enfermería en rehabilitación; estructuras de acceso; personas con discapacidad; limitación de la movilidad

\section{ABSTRACT}

Background: the rehabilitation nurses are decisive to the promotion of accessibility trough architectural barriers elimination, which promotes the reintegration of people with reduced mobility.

Objectives: to understand nurses concernment about the elimination of architectural barriers.

Methodology: a quantitative, descriptive, exploratory study using an accidental non-probabilistic snowball sampling method, composed of 56 nurses. For data collection, an ad hoc self-administered questionnaire was applied, using Google forms, in November 2016.

Results: $60.7 \%$ of nurses don't know whom to adress to eliminate barriers and $58.9 \%$ did not implement any intervention in that sense. Verified significant statistical differences that favor the rehabilitation nurses concerning their knowledge about: specific legislation about acessibility $(\mathrm{p}=0.000)$ and whom to adress to request the elimination of architectural barriers in the community $(p=0.024)$.

Conclusion: the accessibility and the elimination of architectural barriers should be a part of the daily pratice of nurses. 
Descriptors: rehabilitation nursing; architectural accessibility; disabled persons; mobility limitation

Submetido em: 11/11/2018 Aprovado em: 01/12/2018

\section{INTRODUÇÃO}

A influência e o impacto que o ambiente edificado tem nas pessoas estimula-as para a criação de respostas adaptáveis no meio ambiente. Assim, o ambiente afeta o autocuidado sendo que um ambiente adequado permite o desenvolvimento pessoal, a manutenção da independência apesar da capacidade individual, o estabelecimento de objetivos reais e a adaptação do comportamento para atingir esses resultados. $(1,2,3)$

As barreiras arquitetónicas estão presentes nos vários espaços e contextos do nosso dia-a-dia e são consideradas obstáculos edificados no meio urbano ou nos edifícios e que impedem ou dificultam a livre circulação das pessoas que vivenciam alguma incapacidade, transitória ou permanente. ${ }^{(4,5)}$

Num ambiente acessível uma pessoa com mobilidade em cadeira de rodas, cega ou idosa, não demonstra dificuldade, mas qualquer pessoa num ambiente não acessivel pode experienciar mobilidade condicionada. (6)

É fundamental compreender qual a intervenção do enfermeiro de reabilitação na eliminação de barreiras arquitetónicas e promoção de ambientes acessíveis para pessoas com mobilidade condicionada. Segundo o Regulamento de Competências Específicas do Enfermeiro Especialista em Enfermagem de Reabilitação, este profissional "capacita a pessoa com deficiência, limitação da atividade e ou restrição da participação para a reinserção e exercício da cidadania" e "promove a mobilidade, a acessibilidade e a participação social" pela demonstração de conhecimentos sobre legislação específica, sensibilização da comunidade para a adoção de práticas inclusivas, identificação e eliminação de barreiras arquitetónicas, podendo ainda emitir pareceres técnico-científicos sobre estruturas e equipamentos sociais da comunidade. ${ }^{(7)}$

O estudo deste fenómeno não se deve centrar apenas na perspetiva da pessoa com mobilidade condicionada mas, deve ser alargado a outros atores envolvidos neste processo, como os enfermeiros de reabilitação, explorando novos caminhos e numa abordagem holística do processo de reabilitação, intervindo, de igual modo, na dimensão física, mas também em outras dimensões como, a inclusão social das pessoas com mobilidade condicionada. ${ }^{(8)}$

0 enfermeiro de reabilitação tem um papel preponderante na criação de condições que ajudem a pessoa com mobilidade condicionada promovendo a participação na comunidade, favorecendo a sua autonomia e independência.

Com este estudo pretendemos: compreender se a acessibilidade arquitetónica e a eliminação de barreiras arquitetónicas para pessoas com mobilidade condicionada são uma preocupação dos enfermeiros; analisar as diferenças entre os enfermeiros de reabilitação e os outros enfermeiros sobre a acessibilidade arquitetónica.

A finalidade deste estudo passa por sensibilizar os enfermeiros de reabilitação para a promoção da acessibilidade arquitetónica, de forma a alargar a prestação de cuidados ao nível da reinserção na sociedade e o exercício da cidadania.

Face à contextualização questionamo-nos: Será que os enfermeiros de reabilitação têm conhecimentos acrescidos sobre a eliminação de barreiras arquitetónicas?

Tendo como perguntas de investigação:

- Como se posicionam os enfermeiros sobre a promoção da acessibilidade arquitetónica?

- Será que há diferenças entre os enfermeiros de reabilitação e os outros enfermeiros sobre a acessibilidade?

Esta investigação trata-se de um estudo prévio intitulado "Barreiras arquitetónicas - contexto dos enfermeiros" integrado num projeto de investigação mais abrangente sobre a promoção da acessibilidade arquitetónica nas autarquias, para a inclusão de pessoas com mobilidade condicionada e, cujos resultados já se encontram publicados. ${ }^{(9)}$

\section{MÉTODO}

Trata-se de um estudo descritivo, exploratório, com recurso ao paradigma quantitativo.

A população do estudo são os enfermeiros. A técnica de amostragem foi não probabilística acidental em bola de neve, sendo a amostra constituída por 56 enfermeiros. Os critérios de inclusão: enfermeiros que acedam a redes sociais (facebook) e ao email e, a colheita de dados efetuada através de um questionário ad hoc de autopreenchimento com recurso ao formulário Google, no período de Novembro de 2016. A construção do questionário foi realizada a partir da legislação em vigor.

\section{Variáveis consideradas: características} sociodemográficas; conhecimentos sobre legislação com três dimensões a legislação propriamente dita, o processo de ativação dos meios, o início do processo; conhecimento do símbolo internacional de acessibilidade (sim e não); intervenções na prática diária; barreiras arquitetónicas existentes na área de residência dos enfermeiros.

Os dados foram tratados com recurso ao programa de software IBM SPSS, versão 20.0, e utilizada estatística descritiva através do cálculo de frequências absolutas $(N)$, relativas (\%); medidas de tendência central (média e mediana), medidas de dispersão (mínimo, máximo e desvio padrão) e testes não paramétricos. Para a 
análise de dados foi adotado o valor de $p<0,05$ estatisticamente significativo. ${ }^{(10)}$

Ao longo da pesquisa foram cumpridos os padrões éticos exigidos tendo o estudo sido aprovado pela Comissão de Ética da Escola Superior de Enfermagem do Porto (Parecer $\left.n^{\circ} 11 / 2017\right)$. O consentimento informado foi obtido online através do formulário GOOGLE, aquando da resposta ao questionário.

\section{RESULTADOS}

A amostra é constituída por 5 participantes do género masculino $(8,9 \%)$ e 51 do género feminino $(91,1 \%)$ e têm em média 32 anos de idade, com um limite mínimo e máximo de 22 e 63 respetivamente, e um desvio padrão de 7,9 anos.

No que respeita aos anos de exercício profissional em média exercem há 8,9 anos, correspondendo a uma moda de 8 anos e um desvio padrão de 7,869; sendo que no mínimo exercem a função há menos de um ano e no máximo há 37 .

Quanto à formação 44,6\% possuem licenciatura, 21,4\% especialidade em enfermagem de reabilitação, 10,7\% outra especialidade, 3,6\% possuem mestrado em enfermagem de reabilitação, 16,1\% possuem outro mestrado e 3,6\% possuem doutoramento. A maioria desenvolve a sua atuação profissional ao nível hospitalar (76,8\%), 17,9\% em outros locais (como lares, centros de dia, escolas) e 5,4\% na comunidade.

Segundo o teste de normalidade (Teste de KolmogorovSmirnov) verificamos que a distribuição da amostra não é normal, relativamente à idade nem ao tempo de exercício profissional $(p=0)$.

Constatamos que 31 enfermeiros $(55,4 \%)$ têm conhecimento sobre legislação específica para a acessibilidade aos edifícios e estabelecimentos que recebem público, via pública e edifícios habitacionais e 25 não $(44,6 \%)$.
Relativamente ao processo de ativação de meios responsáveis na eliminação das barreiras arquitetónicas existentes para as pessoas com mobilidade condicionada na comunidade 23 enfermeiros $(41,8 \%)$ dizem conhecer e 32 não $(58,2 \%)$ conhece o processo. No caso de precisarem de solicitar alguma intervenção para eliminar barreiras arquitetónicas para pessoas com mobilidade condicionada na comunidade 22 enfermeiros $(39,3 \%)$ sabem a quem se dirigir porém, 34 não sabem $(60,7 \%)$.

Mais de metade dos enfermeiros $(58,9 \%)$ na sua prática diária não desenvolvem qualquer tipo de intervenção no sentido de eliminar barreiras arquitetónicas para as pessoas com mobilidade condicionada mas, $41,1 \%$ dizem que intervêm neste âmbito.

Quanto ao símbolo internacional de acessibilidade $83,6 \%$ conhecem e $16,4 \%$ dos enfermeiros não conhecem.

Da análise do olhar dos enfermeiros sobre as barreiras arquitetónicas existentes no ambiente envolvente à sua área de residência verificamos, conforme a tabela 1, que algumas vezes os passeios e outros percursos pedonais, como rampas e escadas, cumprem o disposto na legislação (87,5\%); as instalações desportivas possuem pelo menos um percurso acessível para pessoas em cadeira de rodas $(57,1 \%)$ e uma cabine de duche adaptada $(58,9 \%)$; as piscinas públicas têm pelo menos um acesso à água por rampa/meio mecânico $(53,7 \%)$; os edifícios públicos têm pelo menos um percurso acessível ao seu interior $(48,2 \%)$ e instalações sanitárias adaptadas em locais públicos como cafés, escolas, supermercados, centro de saúde $(69,6 \%)$.

De salientar que quanto aos lugares de estacionamento para pessoas com mobilidade condicionada $50 \%$ dos enfermeiros referem que existem muitas vezes e $25 \%$ refere existir sempre. Quanto aos passeios e outros percursos pedonais, os enfermeiros nunca referiram o sempre cumprir o disposto na legislação.

\begin{tabular}{|c|c|c|c|c|c|c|c|c|c|c|}
\hline \multirow[t]{2}{*}{ Dimensões } & \multicolumn{2}{|c|}{ Nunca } & \multicolumn{2}{|c|}{$\begin{array}{l}\text { Algumas } \\
\text { vezes }\end{array}$} & \multicolumn{2}{|c|}{$\begin{array}{l}\text { Muitas } \\
\text { vezes }\end{array}$} & \multicolumn{2}{|c|}{ Sempre } & \multicolumn{2}{|c|}{ Total } \\
\hline & $\mathrm{N}$ & $\%$ & $\mathrm{~N}$ & $\%$ & $\mathrm{~N}$ & $\%$ & $\mathrm{~N}$ & $\%$ & $\mathrm{~N}$ & $\%$ \\
\hline $\begin{array}{l}\text { Passeios e outros percursos pedonais cumprem o disposto na } \\
\text { legislação }\end{array}$ & 3 & 5,4 & 49 & 87,5 & 4 & 7,1 & 0 & 0,0 & 56 & 100 \\
\hline $\begin{array}{l}\text { Instalações desportivas possuem pelo menos um percurso } \\
\text { acessivel para pessoa em cadeira de rodas }\end{array}$ & 3 & 5,4 & 32 & 57,1 & 19 & 33,9 & 2 & 3,6 & 56 & 100 \\
\hline $\begin{array}{l}\text { Instalações desportivas possuem pelo menos uma cabine de } \\
\text { duche adaptada }\end{array}$ & 10 & 17,9 & 33 & 58,9 & 10 & 17,9 & 3 & 5,4 & 56 & 100 \\
\hline $\begin{array}{l}\text { Piscinas públicas possuem pelo menos um acesso à água por } \\
\text { rampa/meio mecânico }\end{array}$ & 16 & 29,6 & 29 & 53,7 & 6 & 11,1 & 3 & 5,6 & $54^{*}$ & 96,4 \\
\hline $\begin{array}{l}\text { Edifícios públicos possuem pelo menos um percurso acessível } \\
\text { para o acesso ao seu interior }\end{array}$ & 4 & 7,1 & 27 & 48,2 & 19 & 33,9 & 6 & 10,7 & 56 & 100 \\
\hline $\begin{array}{l}\text { Lugares de estacionamento para pessoas com mobilidade } \\
\text { condicionada }\end{array}$ & 0 & 0,0 & 14 & 25 & 28 & 50 & 14 & 25 & 56 & 100 \\
\hline $\begin{array}{l}\text { Instalações sanitárias adaptadas (cafés, escolas, } \\
\text { supermercados, centro de saúde) }\end{array}$ & 1 & 1,8 & 39 & 69,6 & 15 & 26,8 & 1 & 1,8 & 56 & 100 \\
\hline
\end{tabular}

*Nesta questão ocorreu um missing

Tabela 1 - Opinião sobre as barreiras arquitetónicas mais frequentes no acesso a locais públicos/via pública, na área de residência 
Não se verificaram diferenças estatisticamente significativas relativamente ao conhecimento do processo de ativação dos meios responsáveis para a eliminação das barreiras arquitetónicas existentes para as pessoas com mobilidade condicionada na comunidade $(p=0,281)$; de legislação específica para a acessibilidade aos edifícios e estabelecimentos que recebem público, via pública e edifícios habitacionais através do Decreto-Lei $n^{\circ} 163 / 2006(p=0,504)$; se sabe a quem se dirigir se precisar de solicitar intervenção para eliminar algum tipo de barreira arquitetónica para pessoas com mobilidade condicionada na comunidade $(p=0,142)$ e; no exercício da sua prática diária desenvolve algum tipo de intervenção no sentido de eliminar barreiras arquitetónicas para as pessoas com mobilidade condicionada promovendo a mobilidade, a acessibilidade e a participação $(p=0,229)$.

Porém, há diferenças estatísticas significativas, sendo $p=0,000$, no conhecimento do símbolo internacional de acessibilidade; no cumprimento de disposições legais nos passeios e outros percursos pedonais; as instalações desportivas têm pelo menos um percurso acessível para cadeira de rodas, e têm pelo menos uma cabine de duche adaptada; nas instalações de piscinas públicas há pelo menos um acesso à água por rampa ou por meios mecânicos; nos edifícios da Administração Pública têm pelo menos um percurso acessível para o acesso ao seu interior; nas zonas sociais; nos serviços públicos (cafés, restaurantes, escolas, supermercados, centro de saúde, entre outros) há Instalações sanitárias para pessoas com mobilidade condicionada; e com $p=0,030$ nos parques públicos há lugares de estacionamento para pessoas com mobilidade condicionada.

Depois de analisados os dados no global da amostra foram constituídos dois grupos, enfermeiros com especialidade em enfermagem de reabilitação e os enfermeiros sem especialidade em enfermagem de reabilitação. Assim, 40 enfermeiros não possuem especialidade em enfermagem de reabilitação $(71,4 \%)$ e $16(28,6 \%)$ possuem. Os enfermeiros com especialidade têm em média 33,69 anos de idade, com um desvio padrão de 9,17 anos e, exercem atividade profissional em média há 10,64 anos, com um desvio padrão de 9,54 anos.

Por outro lado, os enfermeiros sem especialidade em enfermagem de reabilitação têm em média 31,48 anos, desvio padrão de 7,44 anos e exercem em média há 8,37 anos com um desvio padrão de 7,22 anos.

Não há associação entre ter ou não ter especialidade em enfermagem de reabilitação e o género $(p=1)$. Há uma associação perfeita entre a formação profissional e o ser ou não enfermeiro de reabilitação $(p=0,000)$.

Não há associação entre o local de trabalho e o ser ou não enfermeiro de reabilitação $(p=0,974)$.

Há uma associação perfeita entre o ser enfermeiro de reabilitação e ter conhecimento sobre legislação específica para a acessibilidade aos edifícios e estabelecimentos que recebem público, via pública e edifícios habitacionais através do Decreto-Lei $n^{\circ} 163 / 2006(p=0,000)$.
Há associação entre o ser enfermeiro de reabilitação e o saber a quem se dirigir se precisar de solicitar intervenção para eliminar algum tipo de barreira arquitetónica para pessoas com mobilidade condicionada na comunidade $(p=0,024)$ e 0 conhecimento do processo de ativação dos meios responsáveis para eliminar as barreiras arquitetónicas existentes para pessoas com mobilidade condicionada na comunidade $(p=0,04)$.

Não há associação entre o ser enfermeiro de reabilitação e no exercício da prática diária desenvolver algum tipo de intervenção no sentido de eliminar barreiras arquitetónicas para as pessoas com mobilidade condicionada promovendo a mobilidade, a acessibilidade e a participação $(p=0,390)$. Não há associação entre o ser enfermeiro de reabilitação e o conhecer o símbolo internacional de acessibilidade $(p=0,710)$.

Não há diferenças estatisticamente significativas entre os dois grupos relativamente à existência das barreiras arquitetónicas na área de residência destes enfermeiros salientando-se: os passeios, rampas e escadas cumprem o disposto na legislação $(p=0,516)$; as instalações desportivas têm um percurso acessível para cadeira de rodas $(p=0,375)$; têm uma cabine de duche adaptada $(p=0,275)$; nas piscinas públicas há acesso à água por rampa/meios mecânicos $(p=0,213)$; os edifícios da Administração Pública têm um percurso acessível no acesso ao interior $(\mathrm{p}=0,775)$; nos locais públicos há lugares de estacionamento para pessoas com mobilidade condicionada $(\mathrm{p}=1)$; nos serviços públicos há instalações sanitárias adaptadas $(p=0,317)$.

\section{DISCUSSÃO}

Questionamo-nos como se posicionam os enfermeiros sobre a promoção da acessibilidade arquitetónica sendo que na realidade dos participantes do nosso estudo mais de metade $(55,4 \%)$ declara que possui conhecimento sobre a legislação específica para a acessibilidade aos edifícios e estabelecimentos que recebem público, via pública e edifícios habitacionais através do Decreto-Lei $\mathrm{n}^{\circ} 163 / 2006$, de 8 Agosto, mas 44,6\% dos enfermeiros não conhece apesar de, no nosso país, o enquadramento legal da acessibilidade e mobilidade para todos nos remeter para o ano de 1997.

Atualmente, vigora o Decreto-Lei no 163/2006 de 8 de Agosto designado por Regime da acessibilidade aos edifícios e estabelecimentos que recebem público, via pública e edifícios habitacionais. (11) Este aconselha a utilização do símbolo internacional de acessibilidade, num local facilmente visto, lido e entendido por qualquer pessoa de pé ou sentada, para que a oriente e direcione para entradas/saídas e percursos acessíveis, bem como identifique lugares de estacionamento reservados para pessoas com mobilidade condicionada e instalações sanitárias acessíveis.

Dos inquiridos 83,6\% conhece este símbolo, contudo, apesar de na legislação ser sugerido este símbolo, a sua utilização ainda não é generalizada; uma vez que num estudo realizado em 27 escolas do município de Chapecó, no qual avaliaram o ambiente externo e 
interno das escolas relativamente às condições de acessibilidade, registaram que em nenhuma escola foi observada a presença deste símbolo. ${ }^{(12)}$

As autarquias locais são uma entidade governativa mais próxima das pessoas e à qual podem recorrer para eliminar algumas barreiras arquitetónicas; logo, têm um grande destaque na promoção de um ambiente acessível e livre de barreiras para todos. (13)

Num estudo realizado em três concelhos da região Centro de Portugal, no qual efetuaram o levantamento documental dos programas de gestão autárquica desses concelhos, encontraram um programa habitacional que tem como objetivo a melhoria das condições de acessibilidade arquitetónica, por exemplo, através da realização de pequenas reparações no interior da habitação. ${ }^{(5)}$ No mesmo estudo também constataram que em algumas autarquias existem programas de intervenção no ambiente e em espaços verdes os quais contemplaram medidas de adaptação dos espaços para pessoas com mobilidade condicionada.

Quando questionados se conhecem o conhecem o processo de ativação dos meios responsáveis para a eliminação das barreiras arquitetónicas existentes para as pessoas com mobilidade condicionada na comunidade $41,8 \%$ dos enfermeiros conhece mas mais de metade $(58,2 \%)$ não conhece. No desenvolvimento da sua prática profissional, se precisar de solicitar intervenção para eliminar algum tipo de barreira arquitetónica para pessoas com mobilidade condicionada na comunidade $60,7 \%$ dos enfermeiros não sabem a quem se dirigir.

Daqui podemos compreender que não há uma articulação entre o setor da saúde e os órgãos autárquicos nesta matéria, apesar de algumas Câmaras possuírem uma Provedoria da Pessoa com Deficiência, Gabinetes de Promoção de Saúde ou Programas de Envelhecimento ativo. (5)

No Relatório das Nações Unidas sobre o direito à habitação e à vida nas cidades para pessoas com deficiência é salientado que as autarquias locais têm extrema relevância no desenvolvimento de planos que garantam o acesso sem barreiras arquitetónicas a serviços e espaços públicos no sentido de promover a vida independente. (14)

No sentido de compreender se os enfermeiros desenvolvem ações que visem a promoção de ambientes acessíveis verificamos que, do total da amostra e, na sua prática diária, mais de metade $(58,9 \%)$ não realiza qualquer intervenção no âmbito da eliminação de barreiras arquitetónicas na comunidade, apesar de "atuar como um recurso para os indivíduos, para as famílias e para as comunidades que enfrentam desafios colocados pela saúde, pela deficiência e pela morte”. (15)

Um dos nossos objetivos era analisar as diferenças entre os enfermeiros com e sem especialidade em enfermagem de reabilitação sobre a acessibilidade arquitetónica, assim questionamo-nos se existem diferenças entre os enfermeiros de reabilitação e os outros enfermeiros sobre a acessibilidade?
De acordo com as competências do enfermeiro de reabilitação ${ }^{(7)}$ é da sua responsabilidade trabalhar para a inclusão das pessoas com mobilidade condicionada mostrando que apesar das limitações funcionais, é possível a sua integração e participação nos diferentes contextos (trabalho, lazer...). Este facto é corroborado pelo nosso estudo que confirma uma associação entre o ser enfermeiro de reabilitação e possuir conhecimento sobre legislação específica para a acessibilidade, o saber a quem se dirigir se precisar de solicitar intervenção para eliminar algum tipo de barreira arquitetónica na comunidade e o conhecimento do processo de ativação dos meios responsáveis para a sua eliminação.

Sendo uma das competências dos enfermeiros de reabilitação a identificação e orientação para a eliminação de barreiras arquitetónicas nos diferentes contextos da pessoa, era expectável que, no exercício da prática diária desenvolvessem algum tipo de intervenção nesse sentido promovendo a mobilidade, a acessibilidade e a participação. Todavia, não se constataram diferenças estatisticamente significativas.

Relativamente às barreiras arquitetónicas existentes na área de residência dos enfermeiros verificamos diferenças estatisticamente significativas nos edifícios da Administração Pública, nos quais, apenas algumas vezes possuem pelo menos um percurso acessível para o acesso ao seu interior; nas zonas sociais. Nos Censos de 2011, referem que "a maioria dos edifícios não era acessível a pessoas com mobilidade condicionada" e que cerca de $59 \%$ dos edifícios, não apresentavam entrada acessível para uma pessoa com mobilidade condicionada. ${ }^{(16)}$

Também os passeios e outros percursos pedonais, como rampas e escadas, apenas algumas vezes cumprem as disposições legais, havendo uma relação estatisticamente significativa, sendo que na via pública os passeios assumem grande importância para as pessoas com mobilidade condicionada e tornam-se barreiras graves para a livre e segura circulação de pessoas devido às suas dimensões inadequadas ou em alguns casos pela sua ausência, bem como, pelo seu estado de conservação, ressaltos no piso e existência de elementos vegetais, mobiliário urbano e a inclinação das rampas que, por vezes, são tão íngremes que têm que contar com a ajuda de terceiros para vencer os desníveis. $(11,17)$

$\mathrm{Na}$ realidade dos participantes, há diferenças estatísticas significativas quanto às instalações desportivas terem ou não terem pelo menos um percurso acessível para cadeira de rodas, e pelo menos uma cabine de duche adaptada; e nas instalações de piscinas públicas existir pelo menos um acesso à água por rampa ou por meios mecânicos.

Sabemos que, a prática regular de atividade física, nas pessoas com deficiência física, evidencia resultados positivos na sua perceção de qualidade de vida. (18) Porém, num estudo que avaliou as principais barreiras e os principais facilitadores percebidos para a prática de atividade física por pessoas com deficiência motora, relativamente aos indicadores relacionados com 0 ambiente e a acessibilidade arquitetónica não 
encontraram diferenças estatisticamente significativas; ou seja, estes fatores não interferem de forma positiva para a prática de atividade física. (19)

Perestrelo ${ }^{(20)}$ assume que é essencial a mobilização em prol do outro desenvolvendo uma cultura democrática e participativa pelo que consideramos importante a existência de um compromisso por parte dos profissionais de saúde, nomeadamente dos enfermeiros de reabilitação, na eliminação de barreiras arquitetónicas através de pedidos e propostas de melhoria das condições de acessibilidade. Já que se verifica um fraco envolvimento destes profissionais na promoção de ambientes acessíveis, nomeadamente na sua área de residência, dado que não há diferenças estatisticamente significativas entre ter e não ter especialidade em enfermagem de reabilitação.

Em suma, e atendendo à pergunta de partida podemos afirmar que os enfermeiros de reabilitação possuem conhecimentos acrescidos relativamente aos outros enfermeiros sobre a eliminação de barreiras arquitetónicas. Todavia, para reforçar os ganhos das pessoas em acessibilidade arquitetónica é fundamental a criação de sinergias, através da identificação e mobilização de redes de atores locais (económicos, políticos, sociais, associativos, entre outros), valorizando as redes da comunidade e outras redes informais. (21)

Os enfermeiros de reabilitação devem ampliar esforços entre os vários parceiros, aproximar a decisão das pessoas e atender às suas necessidades, de acordo com os contextos em que desenvolve o exercício profissional procurando soluções criativas para problemas particulares, como a eliminação de barreiras arquitetónicas na via pública e locais públicos, mas também nas suas casas. Reconhecemos que o meio edificado necessita muitas vezes de ser repensado face à sua utilização por pessoas com mobilidade condicionada.

\section{CONCLUSÃO}

A enfermagem é uma disciplina científica que, habitualmente, tem como ponto central a prestação de cuidados à pessoa; porém o alvo dos cuidados de enfermagem também é a família, um grupo, uma comunidade ou a sociedade.

Pela aplicação do Decreto-Lei no 163/2006, era expectável que em Fevereiro de 2017 não existissem barreiras arquitetónicas em locais de uso público dado terminar o prazo para adaptação dos espaços (edifícios, estabelecimentos e equipamentos de utilização pública cuja data de construção era anterior a 1997). Contudo, os nossos resultados demonstram que ainda subsistem barreiras arquitetónicas nas áreas de residência dos enfermeiros.

Com a realização deste estudo compreendemos que a acessibilidade e a eliminação de barreiras arquitetónicas não são uma preocupação na prestação de cuidados dos enfermeiros uma vez que não se verificaram diferenças estatisticamente significativas relativamente ao conhecimento do processo de ativação dos meios responsáveis para a eliminação das barreiras arquitetónicas; da legislação específica para a acessibilidade; se sabe a quem se dirigir se precisar de solicitar intervenção para eliminar algum tipo de barreira arquitetónica e se no exercício da sua prática diária desenvolve algum tipo de intervenção no sentido de eliminar barreiras arquitetónicas.

Comprovam-se diferenças entre os enfermeiros com e sem especialidade em enfermagem de reabilitação, nomeadamente no conhecimento de legislação específica para a acessibilidade, o saber a quem se dirigir se precisar de solicitar intervenção para eliminar algum tipo de barreira arquitetónica para pessoas com mobilidade condicionada na comunidade e o conhecimento do processo de ativação dos meios responsáveis para eliminar as barreiras arquitetónicas. Mas, não há diferenças relativamente ao desenvolvimento algum tipo de intervenção no sentido de eliminar barreiras arquitetónicas na sua prática profissional.

Os resultados evidenciam uma necessidade de os enfermeiros desenvolverem práticas promotoras da integração e participação cívica pela promoção das condições de acessibilidade.

Dadas as competências dos enfermeiros de reabilitação é inquestionável a sua importância na promoção de ambientes acessíveis. Porém, devem apropriar-se dessas competências e, da sua responsabilidade acrescida enquanto cidadão, envolvendo-se na melhoria das condições de acessibilidade. Acreditamos que é importante para os ganhos em qualidade de vida das pessoas com mobilidade condicionada que, os enfermeiros de reabilitação tenham uma atitude mais proativa nesta área.

Os enfermeiros de reabilitação e os órgãos autárquicos locais podem constituir um binómio de promoção da saúde e da acessibilidade arquitetónica, uma vez que, são os atores políticos de construção de territórios inclusivos, pelo cumprimento de disposições legais, construindo cidades inclusivas para pessoas com mobilidade condicionada.

A progressiva eliminação de barreiras arquitetónicas é essencial para que as pessoas com mobilidade condicionada tenham acesso a todos os sistemas e serviços da comunidade e possam gozar dos seus direitos como cidadãos.

A limitação do estudo está relacionada com o tamanho da amostra que não é representativa do universo dos enfermeiros.

Consideramos ser importante desenvolver outros estudos que avaliem quais as dificuldades que os enfermeiros encontram na sua prática diária quando querem eliminar barreiras arquitetónicas dado que mais de metade reconhece não desenvolver nenhum tipo de intervenção para a eliminação de barreiras arquitetónicas e compreender de que forma se pode potenciar a sua ação na promoção de ambientes acessíveis.

Acreditamos ser fundamental a realização de um estudo sobre a implementação da medida proposta pelo Plano Nacional de Promoção da Acessibilidade relativamente à formação de novos profissionais e a inclusão no plano 
de estudos de módulos de formação sobre a temática da acessibilidade, bem como, repensar a criação de uma rede necessária para serem garantidas as condições de acessibilidade para as pessoas com mobilidade condicionada.

\section{REFERÊNCIAS}

1. Roy C, Andrews HA. Teoria da Enfermagem. O Modelo de Adaptação de Roy. s.l. : Instituto Piaget; 2001.

2. Roper N, Logan W, Tierney AJ. O modelo de enfermagem RoperLogan-Tierney. Lisboa: Climepsi Editores; 2001.

3. Orem, DE. Normas práticas en enfermería. Madrid: Piramide; 1983.

4. Fernandes I, LIPPO H. Política de acessibilidade universal na sociedade contemporânea. Textos e contextos [Internet]. 2013 [acesso em 2017 maio 8]; 12(2): 281-291. Disponível em: http://revistaseletronicas.pucrs.br/ojs/index.php/fass/article/ view/14819/10740

5. Bárrios MJ, Fernandes AA. A promoção do envelhecimento ativo ao nível local: análise de programas de intervenção autárquica. Rev Port Saúde Pública [Internet]. 2014 [acesso em 2017 maio 8]; (12): 188-196. Disponível em: https://ac.elscdn.com/S0870902514000492/1-s2.0-S0870902514000492 main.pdf?_tid=5b3f7885-e506-4fc6-ba963b4b7dbd91 f0\&acdnat=1529432524_9ea4b4fabe7a1f6fe7ec00d25 $4 a 68486$

6. Evcil, AN. Raising awareness about accessibility. Procedia [Internet]. 2012 [acesso em 2017 maio 8]; 47(2012): 490-494. Disponível em: https://ac.els-cdn.com/S1877042812024226/1s2.0-S1877042812024226-main.pdf? tid=e1a09f8d-c07f-40e4a248-

b086de0e8a2d\&acdnat=1529432687_8b24fc9dd83971e7c09d169a $88 f 0 c a 3 a$

7. Portugal. Regulamento n. ${ }^{0} 125 / 2011$, de 18 de Fevereiro de 2011.

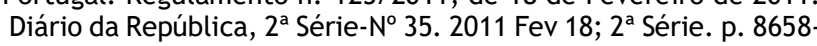
8659.

8. França ISX, Pagliuca LMF. Inclusão social da pessoa com deficiência: conquistas, desafios e implicações para a enfermagem. Rev Esc Enferm USP [Internet]. 2009 [acesso em 2017 maio 16]; 43(1): 178-85. Disponível em: http://www.scielo.br/pdf/reeusp/v43n1/23.pdf

9. Pereira R, Martins M, Gomes B, Schoeller S, Aguilera JL, Ribeiro I et al. As autarquias e a promoção da acessibilidade arquitetónica. Rev Enf Ref. 2018; 18: 29-38.

10. Fortin, MF. Fundamentos e etapas do processo de investigação. Loures: Lusodidacta; 2009.
11. Portugal. Decreto-Lei $n^{\circ} 163 / 2006$ de 8 de Agosto de 2006. Diário da República, $1^{\text {a }}$ Série. № 152. 2006 Agosto 8. p. 5670-5689.

12. Gallo EC, Orso KD, Fiório FB. Análise da acessibilidade das pessoas com deficiência física nas escolas de Chapecó-SC e o papel do fisioterapeuta no ambiente escolar. Mundo Saúde [ Internet]. 2013 [acesso em 2017 maio 16]; 35(2): 201-207. Disponível em: http://bvsms.saude.gov.br/bvs/artigos/analise_acessibilidade_p essoas_deficiencia_fisica_escolas.pdf

13. Cruz CF, Ferreira ACS, Silva LM, Macedo MAS. Transparência da gestão pública municipal: um estudo a partir dos portais eletrónicos dos maiores municípios brasileiros. Rev. Adm. Pública [Internet]. 2012 [acesso em 2017 maio 8]; 46(1): 153-176. Disponível em http: / / www. scielo.br/scielo.php?script=sci arttext\&pid=S003476122012000100008

14. Szporluk M. The Right to Adequate Housing for Persons With Disabilities Living in Cities. Quénia; UN-Habitat, 2015.

15. Portugal. Regulamento n. ${ }^{\circ} 190 / 2015$, de 23 de Abril 2015. Diário da República, $2^{\mathrm{a}}$ Série- $N^{\circ}$ 79. 2015 Abril 23; 2 ${ }^{\mathrm{a}}$ Série. p. 1008710090.

16. INE. Censos 2011 Resultados Definitivos - Portugal. Lisboa; Instituto Nacional de Estatística, 2012.

17. Lima SSC, Carvalho-Freitas MN, Santos LMM. Repercussões psicossociais da acessibilidade urbana para as pessas com deficiência física. Psico [Internet]. 2013 [acesso em 2017 maio 8]; 44(3): $\quad 362-371 . \quad$ Disponível em http://revistaseletronicas.pucrs.br/ojs/index.php/revistapsico/ article/view/15823/10411

18. Noce F, Simim MAM, Mello MT. A percepção de qualidade de vida de pessoas portadoras de deficiência física pode ser influenciada pela prática de atividade física. Rev Bras Med Esporte [Internet]. 2009 [acesso em 2018 Março 15]; 15(3): 174-178. Disponível em http://www.scielo.br/pdf/rbme/v15n3/a02v15n3.pdf

19. Seron BB, Arruda GA, Greguol M. Facilitadores e barreiras percebidas para a prática de atividade física por pessoas com deficiência motora. Rev Bras Ciênc Esporte [Internet]. 2015 [acesso em 2018 Março 15] 37 (3): 214-221. Disponível em http://www.scielo.br/pdf/rbce/v37n3/0101-3289-rbce-37-030214.pdf

20. Perestrelo M. Prospetiva e democracia participativa: sucessos e insucessos. In: Marques AP, Moreira R. Governação e Territorialidades - Lógicas e práticas na administração local. Famalicão: Edições Húmus; 2012. P. 95-11.

21. Rufino AC, Bonfim WLS. Diversas abordagens conceituais sobre o trabalho em rede. Barbarói [Internet]. 2015 [acesso em 2017 maio 8]; $45 \quad$ (2): 119-137. Disponível em https://online.unisc.br/seer/index.php/barbaroi/article/view/4 905/5240. 